\title{
VARIACIÓN ANUAL DEL POLEN EN LA ATMÓSFERA DE PONFERRADA. AÑOS 1995-96.
}

\author{
Ana VEGA MARAY, Rosa $M^{\mathrm{a}}$ VALENCIA BARRERA, \\ Delia FERNÁNDEZ GONZÁLEZ y Juan M. ESTRADA
}

\begin{abstract}
RESUMEN. Variación anual del polen en la atmósfera de Ponferrrada. Años 1995-96. El objeto de este estudio es conocer la concentración de polen y la periodicidad anual del mismo, en la atmósfera de la ciudad de Ponferrada (León). El muestreo aerobiológico se ha realizado durante dos años consecutivos (1995-96), mediante un captador volumétrico tipo Hirst (modelo Lanzoni VPSS-2000), situado a unos $10 \mathrm{~m}$ del suelo. Durante este periodo se han identificado 66 tipos polínicos de ellos, $22(96 \%)$ constituyen el espectro polínico principal, de esta estación de muestreo. Entre Enero y Julio, se produce la máxima emisión de polen al aire, y desde Septiembre a Diciembre la cantidad de polen atmósferico es muy escasa. Los tipos polínicos predominantes cuantitativamente han sido: Cupressaceae $(21,3 \%)$, Poaceae $(19,2 \%)$, Pinaceae $(12,6 \%)$, Quercus (10\%), Castanea sativa (6,2\%), y Betula (3,6\%), que junto con Alnus, Erica, Plantago, Populus, Rumex y Urticaceae, suponen el $91,9 \%$ del polen total de la atmósfera de Ponferrada. Se ha detectado un predominio anual de pólenes de especies leñosas frente a herbáceas. Desde el punto de vista clínico, los pólenes de Poaceae $(60 \%)$ son, con mucho, los que ocasionan mayor patología polínica, seguidos de Plantago (27\%) y Betula (12\%).
\end{abstract}

Palabras clave. Aerobiología, Polen, Ponferrada, NW de España.

ABSTRACT. Pollen annual variation in the atmosphere of Ponferrada. Years 1995-96. The aim of this study is to know the pollen concentration and its annual periodicity in the atmosphere of the city of Ponferrada (León, NW Spain). The aerobiological sampling was carried out using a Lanzoni VPSS-2000 sporetrap located about $10 \mathrm{~m}$ above ground level. Samples were collected in two consecutive years, 1995 and 1996. We have identified 66 different pollinic types for this sampling period but $96 \%$ of the samples corresponded to only 22 types which formed the main pollinic spectrum for this sampling station. The amount of atmospheric pollen from September to December was very scarce and the maximum of pollen emissions was produced between January and July. The predominant pollinic types were: Cupressaceae (21,3\%), Poaceae (19,2\%), Pinaceae (12,6\%), Quercus (10\%), Castanea sativa $(6,2 \%)$, and Betula $(3,6 \%)$; these six types plus Alnus, Erica, Plantago, Populus, Rumex and Urticaceae included the $91,9 \%$ of the pollen present in the atmosphere of Ponferrada. Pollen from woody species was present in higher proportion that pollen from herbaceous species. Clinic data revealed that the Poaceae pollens cause the main pollinic pathologies (60\%) in the city of Ponferrada, followed by Plantago (27\%), and Betula (12\%) pollens.

Key words. Aerobiology, Pollen, Ponferrada, NW Spain.

\section{INTRODUCCIÓN}

La ciudad de Ponferrada está situada al NW de la Península Ibérica a $42^{\circ} 33^{\prime}$ de latitud
$\mathrm{N}$ y $6^{\circ} 35^{\prime}$ de longitud $\mathrm{W}$ y a una altitud de 541 m.s.n.m. Se encuentra en el cuadrante suroccidental de la provincia de León, enclavada en el valle de El Bierzo, limitado 
por los montes Galaico-Astúricos al N y NW y por los Montes de León al S, dando lugar a la formación de la "cubeta berciana", constituida principalmente por terrazas del Cuaternario, asentadas sobre materiales terciarios y paleozoicos.

Ponferrada tiene un clima templado esencialmente húmedo, con oscilaciones elevadas de temperatura y con poca insolación. Su temperatura media anual es de $12,9^{\circ} \mathrm{C}$, con medias estacionales que oscilan desde $21^{\circ} \mathrm{C}$ en verano hasta $5,2{ }^{\circ} \mathrm{C}$ durante el invierno. Esta localidad al estar rodeada de montañas, se halla protegida de los vientos fríos del N, sin embargo, está expuesta a los de dirección W y NW que, procedentes de Galicia, penetran por el valle del Miño y luego por su afluente el Sil, por esta razón, la nubosidad es abundante. Las precipitaciones son numerosas entre los meses de Septiembre y Enero, la cantidad anual es de $640 \mathrm{~mm}$. Las nieblas son relativamente frecuentes, se observan durante el $5 \%$ de los días del año. La humedad relativa media es del $70 \%$.

Ponferrada y su entorno, enclavados biogeográficamente en el Sector OrensanoSanabriense de la Provincia Carpetano-IbéricoLeonesa de la Región Mediterránea, corresponden, desde el punto de vista bioclimático, a territorios de termotipo mesomediterráneo superior y supramediterráneo inferior y de ombrotipo subhúmedo inferior o medio.

Bajo esas condiciones climáticas la vegetación potencial del territorio se corresponde con las series de vegetación de los encinares de la Genisto hystricis-Querceto rotundifoliae sigmetum y de los melojares subhúmedos de la Genisto falcatae-Querceto pyrenaicae sigmetum. En los fondos de los valles de los ríos se detecta la geoserie riparia constituida por las series de las olmedas: Aro maculati-Ulmeto minoris sigmetum, de las choperas-saucedas arbóreas: Populo nigraeSaliceto neotrichae sigmetum, de las alisedas: Galio broteriani-Alneto glutinosae sigmetum y de las saucedas arbustivas: Saliceto lambertiano-angustifoliae sigmetum y Saliceto angustifolio-salvifoliae sigmetum.

Por todo ello, en los alrededores de la ciudad de Ponferrada nos podemos encontrar, en las etapas maduras de dichas series, con especies arbóreas y arbustivas como: Quercus rotundifolia, Q. pyrenaica, Alnus glutinosa, Populus nigra, Ulmus minor, Fraxinus angustifolia, Corylus avellana, Prunus avium y Salix neotricha.

Son frecuentes los matorrales de degradación de los bosques, como aulagares con Genista hystrix, piornales con Cytisus multiflorus, Genista florida subsp. polygaliphylla, Genistella tridentata, y brezales con Erica umbellata, E. cinerea, E. australis subsp. aragonensis, así como otros taxones: Rosa corymbifera, Rubus ulmifolius, Ligustrum vulgare, Crataegus monogyna, Prunus spinosa, etc.

También son particularmente frecuentes los prados y pastizales en los que participan gramíneas, leguminosas, plantagináceas y compuestas.

En los márgenes de las carreteras y caminos es frecuente, en ambos casos, la aparición de herbazales ruderales de la Artemisietea vulgaris en los que predominan Artemisia vulgaris, Echium vulgare, Lactuca virosa, Reseda luteola, Hordeum murinum, Capsella bursa-pastoris y Achillea millefolium, entre otras.

La flora ornamental está constituida principalmente por falsas acacias (Robinia pseudoacacia), que componen la vegetación principal de los paseos junto con los plátanos de sombra (Platanus hybrida). Además son muy abundantes las mimosas (Acacia sp.) y los chopos y olmos en lugares húmedos. También destacan las coníferas (abetos, cedros, cipreses, pinos, etc.) de gran resistencia a las variaciones climáticas, así como un buen número de arbustos formando setos: aligustre (Ligustrum sp.), boj (Buxus sempervirens), adelfas (Nerium oleander), etc. 
Un número elevado de individuos de este núcleo de población presenta alergias respiratorias importantes, por ello es necesario catalogar y cuantificar los distintos tipos polínicos a fin de determinar cuales son los responsables de dichos problemas. Una vez que se disponga de datos, obtenidos de periodos suficientemente largos, se estará en condiciones de tratar el problema de forma preventiva.

\section{MATERIAL Y MÉTODOS}

Este estudio se llevó a cabo durante 2 años consecutivos, a partir del 31 de Enero de 1995 hasta el 31 Diciembre de 1996. El muestreo polínico se ha realizado mediante un captador volumétrico tipo Hirst (modelo Lanzoni VPPS-2000), situado en el recinto de las piscinas municipales a una altura de unos $10 \mathrm{~m}$ del suelo, sin obstáculos próximos que impidan la llegada de masas de aire. Dicho recinto se encuentra en el casco urbano, en la zona media de la ciudad. En sus alrededores crece una flora ornamental, que hace que algunos taxones puedan estar supravalorados, esto se comentará en otro apartado de este artículo.

La toma de muestras se efectuó semanalmente. El procedimiento y materiales utilizados son los recomendados por el fabricante del aparato, es decir, como material adhesivo se ha utilizado aceite de silicona y como método de montaje glicerogelatina. Para llevar a cabo el recuento de los pólenes se ha seguido la metodología estandarizada para la R.E.A., (Domínguez et al.,1991). Los datos obtenidos se expresan en número de granos de polen por metro cúbico de aire.

El periodo polínico principal (p.p.p.), se ha definido siguiendo los criterios de Nilsson \& Pearsson (1981).

Los parámetros meteorológicos nos han sido suministrados por el Centro Meteorológico Territorial de Castilla y León (estación de
Ponferrada). Los valores de temperaturas máxima, mínima y precipitación, se reflejan en las figuras 1B y 1C. La estación meteorológica está situada a unos $200 \mathrm{~m}$ del lugar de ubicación del aparato de muestreo.

Las pruebas clínicas se realizaron en el Hospital de El Bierzo, en un grupo de 139 pacientes asmáticos alérgicos, con un rango de edad entre 8 y 14 años. Su estudio se realizó mediante prick-test en la región volar del antebrazo, con controles de histamina (+) y suero fisiológico (-). Se consideraron positivas las pápulas con diámetro superior a $3 \mathrm{~mm}$.

\section{RESULTADOS Y DISCUSIÓN}

\section{Características polínicas}

En la atmósfera de Ponferrada, durante los dos años de muestreo, se han identificado un total de 27.346 granos de polen $/ \mathrm{m}^{3}$ de aire y 66 tipos polínicos. De ellos, 11.337 granos $/ \mathrm{m}^{3}$ y 63 tipos correspondieron al año 1995 y 16.009 granos $/ \mathrm{m}^{3}$ y 52 tipos polínicos al año 1996.

La mayor parte del polen total anual se recogió durante los meses de Febrero $(18,6 \%)$, Abril $(23,2 \%)$ y Junio $(19,7 \%)$ en 1995 y en los meses de Marzo (17\%), Mayo (21\%) y Junio (26\%) en 1996 (tabla 1). La frecuencia estacional de los pólenes en la atmósfera de la ciudad muestra que 4 tipos polínicos alcanzan su concentración máxima durante el invierno: Alnus, Cupressaceae, Fraxinus y Populus, 8 en la primavera: Betula, Ericaceae, Pinaceae, Plantago, Platanus, Poaceae, Quercus y Rumex, y 4 en el verano: Artemisia, Castanea, Chenopodiaceae y Urticaceae. En estas latitudes, el otoño es una época en la cual la presencia de polen en el aire es escasa (fig. 1A). Ésto es debido al agostamiento de las plantas herbáceas, al incremento de las precipitaciones, al descenso de las temperaturas y también a la aparición de las primeras heladas.

En la tabla 2 se reflejan, por orden alfabético, los tipos de polen cuantitativamente 


\begin{tabular}{|c|c|c|c|c|c|c|c|c|c|c|c|c|c|c|c|}
\hline & Año & $\mathbf{E}$ & $\mathbf{F}$ & $\mathbf{M}$ & $\mathbf{A}$ & $\mathbf{M}$ & Jn & $\mathrm{Jl}$ & $\mathbf{A}$ & $\mathbf{S}$ & 0 & $\mathbf{N}$ & $\mathbf{D}$ & Total & $\%$ \\
\hline \multirow[t]{2}{*}{ Alnus } & 1995 & - & 75 & 1 & 0 & 0 & 0 & 0 & 0 & 0 & 0 & 0 & 19 & 95 & 0,8 \\
\hline & 1996 & 440 & 202 & 26 & 5 & 0 & 0 & 0 & 0 & 0 & 0 & 0 & 38 & 712 & 4,4 \\
\hline \multirow[t]{2}{*}{ Artemisia } & 1995 & - & 0 & 0 & 0 & 0 & 0 & 0 & 7 & 76 & 4 & 0 & 0 & 88 & 0,8 \\
\hline & 1996 & 0 & 0 & 0 & 0 & 0 & 0 & 1 & 11 & 52 & 3 & 1 & 0 & 69 & 0,4 \\
\hline \multirow[t]{2}{*}{ Asteraceae* } & 1995 & - & 0 & 0 & 3 & 5 & 11 & 3 & 8 & 2 & 1 & 0 & 0 & 33 & 0,3 \\
\hline & 1996 & 0 & 0 & 0 & 0 & 2 & 30 & 15 & 9 & 3 & 1 & 0 & 0 & 60 & 0,4 \\
\hline \multirow[t]{2}{*}{ Betula } & 1995 & - & 0 & 9 & 435 & 7 & 0 & 0 & 0 & 0 & 0 & 0 & 0 & 451 & 4,0 \\
\hline & 1996 & 0 & 0 & 0 & 466 & 40 & 3 & 0 & 0 & 0 & 0 & 0 & 0 & 508 & 3,2 \\
\hline \multirow[t]{2}{*}{ Brassicaceae } & 1995 & - & 0 & 1 & 1 & 12 & 5 & 0 & 1 & 0 & 0 & 0 & 0 & 21 & 0,2 \\
\hline & 1996 & 0 & 0 & 0 & 0 & 19 & 5 & 0 & 2 & 0 & 0 & 0 & 0 & 28 & 0,2 \\
\hline \multirow[t]{2}{*}{ Castanea sativa } & 1995 & - & 0 & 0 & 0 & 1 & 350 & 320 & 21 & 11 & 2 & 0 & 0 & 706 & 6,2 \\
\hline & 1996 & 0 & 0 & 0 & 0 & 0 & 728 & 255 & 7 . & 0 & 0 & 0 & 0 & 990 & 6,2 \\
\hline \multirow{2}{*}{ Chenopodiaceae } & 1995 & - & 0 & 0 & 0 & 0 & 11 & 29 & 60 & 19 & 7 & 0 & 0 & 128 & 1,1 \\
\hline & 1996 & 0 & 0 & 0 & 0 & 0 & 20 & 18 & 31 & 14 & 1 & 0 & 0 & 85 & 0,5 \\
\hline \multirow[t]{2}{*}{ Corylus } & 1995 & - & 25 & 6 & 0 & 0 & 0 & 0 & 0 & 0 & 0 & 0 & 0 & 32 & 0,3 \\
\hline & 1996 & 9 & 8 & 27 & 0 & 0 & 0 & 0 & 0 & 0 & 0 & 0 & 1 & 46 & 0,3 \\
\hline \multirow[t]{2}{*}{ Cupressaceae } & 1995 & - & 1834 & 405 & 41 & 4 & 7 & 3 & 0 & 0 & 6 & 0 & 3 & 2303 & 20,3 \\
\hline & 1996 & 13 & 1343 & 2151 & 15 & 11 & 5 & 0 & 2 & 0 & 6 & 0 & 1 & 3548 & 22,2 \\
\hline \multirow{2}{*}{ Erica } & 1995 & - & 0 & 21 & 234 & 72 & 42 & 1 & 4 & 0 & 0 & 0 & 0 & 375 & 3,3 \\
\hline & 1996 & 0 & 0 & 5 & 192 & 220 & 99 & 9 & 1 & 1 & 1 & 1 & 0 & 529 & 3,3 \\
\hline \multirow[t]{2}{*}{ Fabaceae** } & 1995 & - & 0 & 0 & 10 & 11 & 18 & 5 & 0 & 0 & 0 & 0 & 0 & 44 & 0,4 \\
\hline & 1996 & 0 & 0 & 0 & 0 & 7 & 25 & 9 & 0 & 0 & 1 & 2 & 0 & 45 & 0,3 \\
\hline \multirow[t]{2}{*}{ Fraxinus } & 1995 & - & 15 & 17 & 14 & 0 & 0 & 0 & 0 & 0 & 0 & 0 & 0 & 47 & 0,4 \\
\hline & 1996 & 26 & 0 & 0 & 27 & 5 & 0 & 0 & 0 & 0 & 0 & 0 & 1 & 60 & 0,4 \\
\hline \multirow[t]{2}{*}{ Oleaceae*** } & 1995 & - & 0 & 0 & 15 & 7 & 0 & 0 & 0 & 0 & 0 & 0 & 0 & 23 & 0,2 \\
\hline & 1996 & 0 & 0 & 0 & 12 & 15 & 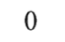 & 0 & 0 & 0 & 0 & 0 & 0 & 27 & 0,2 \\
\hline \multirow[t]{2}{*}{ Pinaceae } & 1995 & - & 36 & 86 & 768 & 285 & 89 & 5 & 4 & 1 & 3 & 1 & 0 & 1279 & 11,3 \\
\hline & 1996 & 0 & 0 & 82 & 823 & 943 & 250 & 20 & 5 & 2 & 60 & 22 & 0 & 2210 & 13,8 \\
\hline \multirow[t]{2}{*}{ Plantago } & 1995 & - & 0 & 3 & 78 & 122 & 105 & 145 & 68 & 14 & 5 & 3 & 0 & 543 & 4,8 \\
\hline & 1996 & 0 & 0 & 0 & 65 & 115 & 88 & 36 & 36 & 12 & 1 & 0 & 0 & 354 & 2,2 \\
\hline \multirow[t]{2}{*}{ Platanus } & 1995 & - & 0 & 22 & 62 & 2 & 0 & 0 & 0 & 0 & 0 & 0 & 0 & 86 & 0,8 \\
\hline & 1996 & 0 & 9 & 58 & 92 & 9 & 0 & 0 & 0 & 0 & 0 & 0 & 0 & 168 & 1,1 \\
\hline \multirow[t]{2}{*}{ Poaceae } & 1995 & - & 31 & 94 & 109 & 279 & 1170 & 505 & 86 & 11 & 6 & 2 & 3 & 2297 & 20,3 \\
\hline & 1996 & 5 & 0 & 0 & 102 & 448 & 1975 & 328 & 23 & 13 & 3 & 2 & 1 & 2899 & 18,1 \\
\hline Populus & 1995 & - & 68 & 385 & 26 & 0 & 0 & 0 & 0 & 0 & 0 & 0 & 0 & 479 & 4,2 \\
\hline & 1996 & 0 & 0 & 298 & 52 & 0 & 0 & 0 & 3 & 3 & 0 & 0 & 0 & 357 & 2,2 \\
\hline Quercus & 1995 & - & 0 & 0 & 624 & 322 & 62 & 6 & 2 & 0 & 0 & 0 & 1 & 1017 & 9,0 \\
\hline & 1996 & 0 & 0 & 0 & 272 & 1117 & 345 & 16 & 4 & 0 & 1 & 1 & 0 & 1756 & 11,0 \\
\hline Rumex & 1995 & - & 0 & 0 & 31 & 113 & 164 & 17 & 7 & 0 & 1 & 0 & 0 & 335 & 3,0 \\
\hline & 1996 & 0 & 0 & 0 & 67 & 299 & 218 & 11 & 2 & 2 & 1 & 0 & 0 & 600 & 3,7 \\
\hline Salix & 1995 & - & 23 & 26 & 37 & 3 & 1 & 0 & 0 & 0 & 0 & 0 & 0 & 90 & 0,8 \\
\hline & 1996 & 0 & 0 & 0 & 31 & 15 & 1 & 0 & 0 & 0 & 0 & 0 & 0 & 47 & 0,3 \\
\hline Urticaceae & 1995 & - & 0 & 7 & 0 & 26 & 127 & 138 & 135 & 5 & 3 & 0 & 0 & 442 & 3,9 \\
\hline & 1996 & 0 & 0 & 0 & 29 & 23 & 157 & 54 & 55 & 9 & 3 & 4 & 0 & 337 & 2,1 \\
\hline Otros & 1995 & - & 3 & 15 & 137 & 69 & 70 & 67 & 49 & 7 & 3 & 0 & 0 & 420 & 3,7 \\
\hline & 1996 & 3 & 19 & 64 & 90 & 66 & 221 & 76 & 21 & 10 & 1 & 2 & 2 & 576 & 3,6 \\
\hline Total & 1995 & - & 2112 & 1097 & 2626 & 1341 & 2233 & 1244 & 454 & 149 & 43 & 8 & 27 & 11337 & 100 \\
\hline & 1996 & 496 & 1582 & 2715 & 2343 & 3354 & 4170 & 848 & 214 & 121 & 83 & 36 & 47 & 16009 & 100 \\
\hline
\end{tabular}

Tabla 1. Sumas mensuales y anuales de las concentraciones medias diarias del polen (granos de polen $/ \mathrm{m}^{3}$ ) y porcentajes (\%) obtenidos para los distintos taxones y para el total de cada mes, durante los años 1995 y 1996. (*excluido Artemisia, **excluido Acacia, ***excluido Fraxinus). Monthly and annual sum of the daily average concentrations (grains $/ \mathrm{m}^{3}$ ) and percentages for the different taxa during 1995 and 1996 (*excluded Artemisia, ** excluded Acacia, ***excluded Fraxinus). 

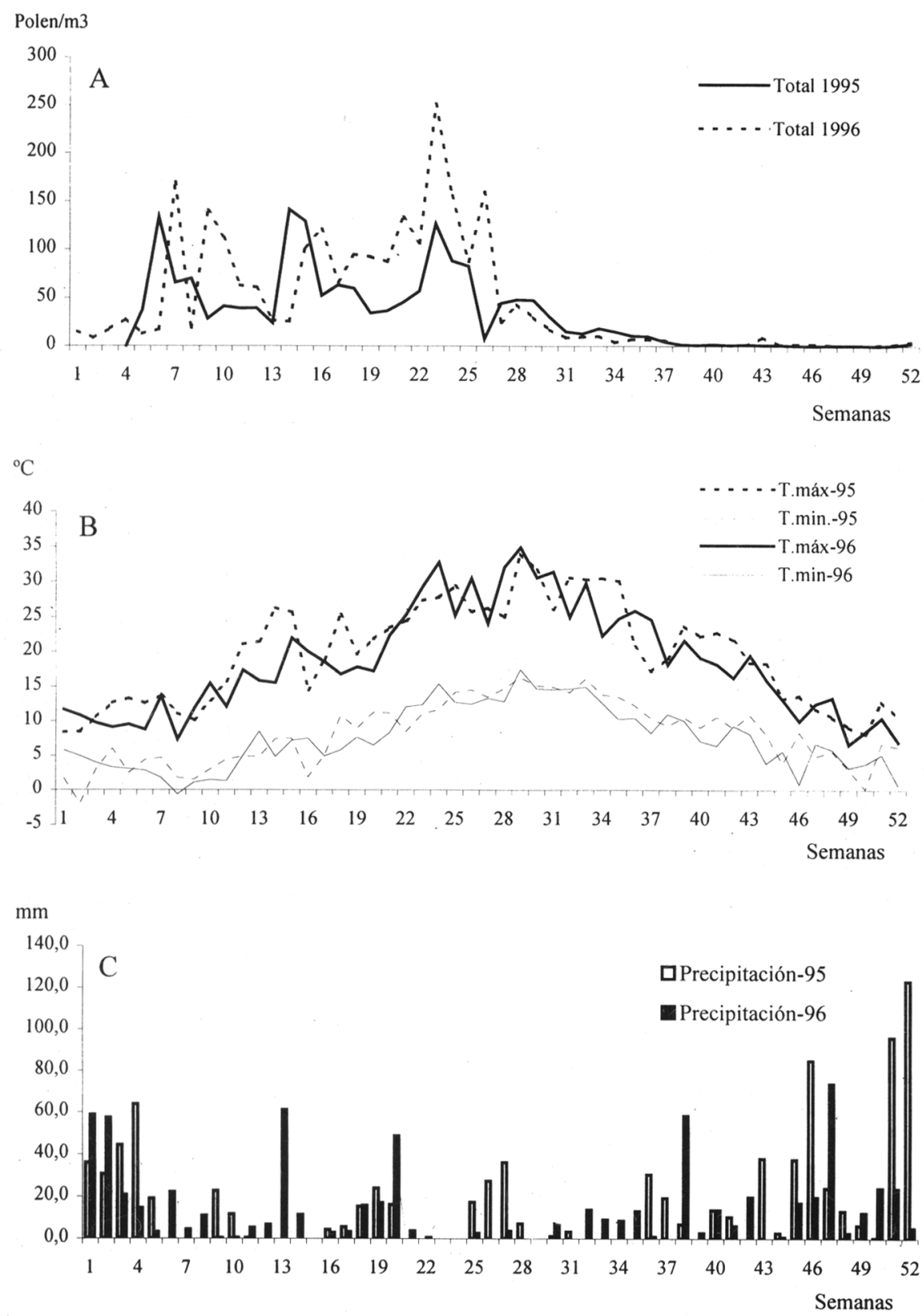

Figura 1. A: Concentración media semanal del polen total registrado en la atmósfera de Ponferrada, durante los años 1995 y 1996, B: Media de las temperaturas máximas semanales durante los años 1995 y 1996 y C: Precipitaciones totales semanales durante los años 1995 y 1996. A: Weekly total pollen concentration trend in the atmosphere of Ponferrada during 1995 and 1996. B: Weekly mean of maximum and minimum temperatures during 1995 and 1996. C: Weekly total rainfalls during 1995 and 1996. 
más abundantes y que más influyen en las polinosis, así como sus valores máximos diarios y semanales. Este grupo representó el 96,3\% del polen total durante el año 1995, el 96,4\% en el año 1996 y es una buena muestra de la vegetación local. Del conjunto de los tipos polínicos identificados, el 63,9\% en 1995 y el $71,1 \%$ en 1996 , correspondieron al polen de taxones arbóreos y arbustivos, siendo inferiores los porcentajes de taxones herbáceos, $36 \%$ en 1995 y $28,9 \%$ en 1996. Esto constituye una importante diferencia con ciudades próximas como León (Fernández-González et al., 1998) y Palencia (Herrero \& Fraile, 1997) de termotipo supramediterráneo. Según Belmonte (1988) y Morales et al. (1998), el predominio de polen de taxones herbáceos frente al polen de taxones arbóreos, caracteriza el calendario polínico de ciudades de interior, mientras que en las ciudades costeras el espectro arbóreo es mayoritario. En nuestro caso, esto no es así, debido posiblemente a las características bioclimáticas de esta región.

La cantidad y la distribución anual de las precipitaciones (fig. 1B), han sido la principal causa de las diferencias polínicas que se han producido durante los dos años de muestreo. Así, el incremento del porcentaje del polen de taxones arbóreos en 1996, ha sido debido a la menor cantidad de precipitaciones caídas entre los meses de Febrero y Marzo. Por el contrario, ese mismo año se produjo un importante descenso cuantitativo de tipos polínicos herbáceos, como consecuencia de las escasas lluvias registradas los meses de Junio y Julio. Ésto, junto con un ligero aumento de las temperaturas medias en este mismo periodo, provocaron un agostamiento precoz de muchos taxones herbáceos.

La dinámica de los pólenes, que representan el espectro polínico principal de la ciudad, con porcentajes superiores al $3 \%$ se indica a continuación.

El polen de Alnus junto con el de la familia Cupressaceae son los más abundantes en la atmósfera de la ciudad durante el periodo invernal. La polinización de Alnus comienza a finales de Diciembre y finaliza a finales de Marzo (fig. 2). Los distintos taxones ornamentales de cupresáceas empiezan su floración a finales del mes de Enero y se prolonga hasta las últimas semanas de Marzo (fig. 2), con un p.p.p. de unas 8 semanas. Hacia mediados del mes de Febrero se registran los niveles polínicos máximos de dicha familia, superando algunos días los 500 granos $/ \mathrm{m}^{3}$. Posteriormente su presencia en la atmósfera es escasa y corresponde a taxones silvestres. Concentraciones similares se han podido observar tanto en ciudades del norte como Santiago de Compostela (Aira et al., 1998), como en la costa mediterránea del sur peninsular (Recio et al., 1996; Toro et al., 1997). No obstante tenemos que destacar la hiperrepresentatividad de este grupo, ya que numerosos ejemplares de cipreses están rodeando la estación captadora. Por ello, su incidencia en las polinosis debe tenerse en cuenta con ciertas reservas.

Los pólenes de Pinaceae siguen en importancia numérica $(12,6 \%)$ y también en orden de aparición en la atmósfera de la localidad, su polinización se observa en periodos pre-primaverales y primaverales, con un p.p.p. de unas 10 semanas. Su presencia comienza a finales de Febrero y se prolonga hasta bien entrado el mes de Julio (fig. 2). Durante los meses de Abril y Mayo se alcanzan las concentraciones más elevadas, llegando a 131 granos $/ \mathrm{m}^{3}$ el día 5 de mayo de 1996.

La polinización de Populus es de tipo explosivo, es decir, corta y relativamente intensa (5 ó 6 semanas). Las concentraciones más altas de este taxon se observan en el mes de Marzo (fig. 3), alcanzando sus valores máximos a mediados de dicho mes, 45 granos/ $\mathrm{m}^{3}$ se registraron el día 17 de Marzo de 1995

El comportamiento estacional, así como la cantidad de polen emitido por Pinaceae y Populus es similar al observado en ciudades 


\begin{tabular}{|c|c|c|c|c|c|}
\hline & \multirow[b]{2}{*}{ Año } & \multicolumn{2}{|c|}{ Máximo diario } & \multicolumn{2}{|c|}{ Media semanal máxima } \\
\hline & & Granos $/ \mathrm{m}^{3}$ & Día & Granos $/ \mathrm{m}^{3}$ & Semana \\
\hline \multirow[t]{2}{*}{ Polen total } & 1995 & 337 & 12-febrero & 141 & 14 (Abril) \\
\hline & 1996 & 594 & 18-Febrero & 251 & 23 (Junio) \\
\hline \multirow[t]{2}{*}{ Alnus } & 1995 & 21 & 6-Febrero & 6 & 6 (Febrero) \\
\hline & 1996 & 51 & 27-Enero & 26 & 4 (Enero) \\
\hline \multirow[t]{2}{*}{ Betula } & 1995 & 87 & 8-Abril & 41 & 14 (Abril) \\
\hline & 1996 & 89 & 12-Abril & 41 & 15 (Abril) \\
\hline \multirow[t]{2}{*}{ Castanea sativa } & 1995 & 64 & 22-Junio & 26 & 25 (Junio) \\
\hline & 1996 & 74 & 27-Junio & 56 & 26 (Junio) \\
\hline \multirow[t]{2}{*}{ Cupressaceae } & 1995 & 329 & 12-Febrero & 122 & 6 (Febrero) \\
\hline & 1996 & 581 & 18-Febrero & 157 & 7 (Febrero) \\
\hline \multirow[t]{2}{*}{ Ericaceae } & 1995 & 30 & 13-Abril & 15 & 15 (Abril) \\
\hline & 1996 & 27 & 23-Мауо & 13 & 17 (Abril) \\
\hline \multirow[t]{2}{*}{ Pinaceae } & 1995 & 119 & 3-Abril & 60 & 14 (Abril) \\
\hline & 1996 & 131 & 5-Mayo & 57 & 16 (Abril) \\
\hline \multirow[t]{2}{*}{ Plantago } & 1995 & 15 & 29-Mayo & 8 & 22 (Junio) \\
\hline & 1996 & 15 & 4-Mayo & 7 & 23 (Junio) \\
\hline \multirow[t]{2}{*}{ Poaceae } & 1995 & 84 & 6-Junio & 100 & 23 (Junio) \\
\hline & 1996 & 114 & 3-Junio & 84 & 23 (Junio) \\
\hline \multirow[t]{2}{*}{ Populus } & 1995 & 45 & 17-Marzo & 22 & 11 (Marzo) \\
\hline & 1996 & 37 & 26-Marzo & 19 & 13 (Marzo) \\
\hline \multirow[t]{2}{*}{ Quercus } & 1995 & 100 & 18-Abril & 38 & 15 (Abril) \\
\hline & 1996 & 134 & 14-Mayo & 52 & 21 (Mayo) \\
\hline \multirow[t]{2}{*}{ Rumex } & 1995 & 17 & 5-Junio & 14 & 23 (Junio) \\
\hline & 1996 & 35 & 4-Mayo & 25 & 23 (Junio) \\
\hline \multirow[t]{2}{*}{ Urticaceae } & 1995 & 16 & 18-Junio & 8 & 24 (Junio) \\
\hline & 1996 & 17 & 16-Junio & 12 & 24 (Junio) \\
\hline
\end{tabular}

Tabla 2. Valores máximos diarios y semanales, de los tipos polínicos más relevantes en la atmósfera de Ponferrada durante los años 1995 y 1996. Daily and weekly maximum values obtained by the most important taxa in the atmosphere of Ponferrada during 1995 and 1996.

próximas como León (Fernández González et al., 1998) y Orense (Iglesias et al., 1998).

El polen de Betula es producido casi exclusivamente por individuos autóctonos, ampliamente representados en esta zona geográfica denominada "cubeta berciana". Aparece durante el mes de Abril, pudiendo prolongarse hasta finales de Mayo (fig. 2), su p.p.p. oscila entre 4 y 7 semanas en función de las condiciones climáticas de años precedentes (Frenguelli et al., 1991, Troise et al., 1992, Dahl \& Strandhede, 1996). Los valores suelen ser medios, aunque algunos días se han llegado a superar los 80 granos $/ \mathrm{m}^{3}$ de aire como ocurre en ciudades de abundantes precipitaciones (Aira et al., 1998 e Iglesias et al., 1998).

El polen de encinas, melojos y carballos (Quercus), cuantitativamente (10\%) es uno de los más importantes. Comienza a detectarse en las muestras a mediados de Abril y se prolonga de forma constante hasta finales de Junio (fig. 3 ), con un periodo de polinización de unas 10 semanas. Entre la segunda quincena de Abril y la primera de Mayo, alcanzan niveles muy altos (más de 100 granos $/ \mathrm{m}^{3}$ ). Se han citado ritmos de producción de este polen de 2 ó 5 años (Emberlin et al., 1990), sin embargo, este hecho no podemos demostrarlo por el momento en este estudio, ya que se necesitan periodos de análisis más largos.

El grupo entomófilo de las Ericaceae contribuye al espectro polínico del aire de Ponferrada con un 3,3\% del contenido polínico global. Está presente durante una buena parte del año, de Marzo a Junio (fig. 2), debido a las sucesivas floraciones de los brezales que rodean 

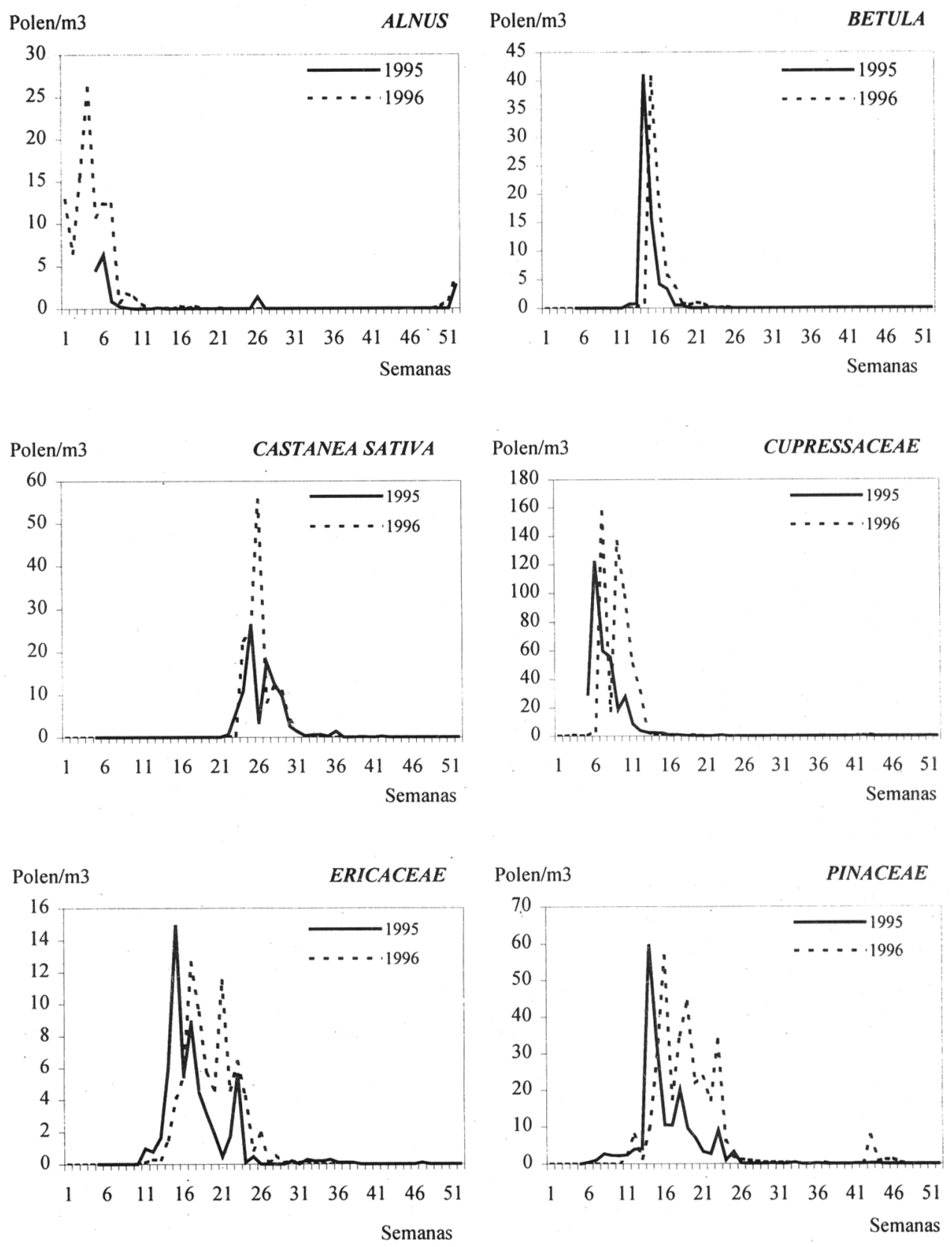

Figura 2. Evolución de las concentraciones medias semanales del polen de Alnus, Betula, Castanea, Cupressaceae, Ericaceae y Pinaceae, en la atmósfera de Ponferrada. Weekly average pollen concentration trend of the Alnus, Betula, Castanea, Cupressaceae, Ericaceae and Pinaceae in the atmosphere of Ponferrada. 

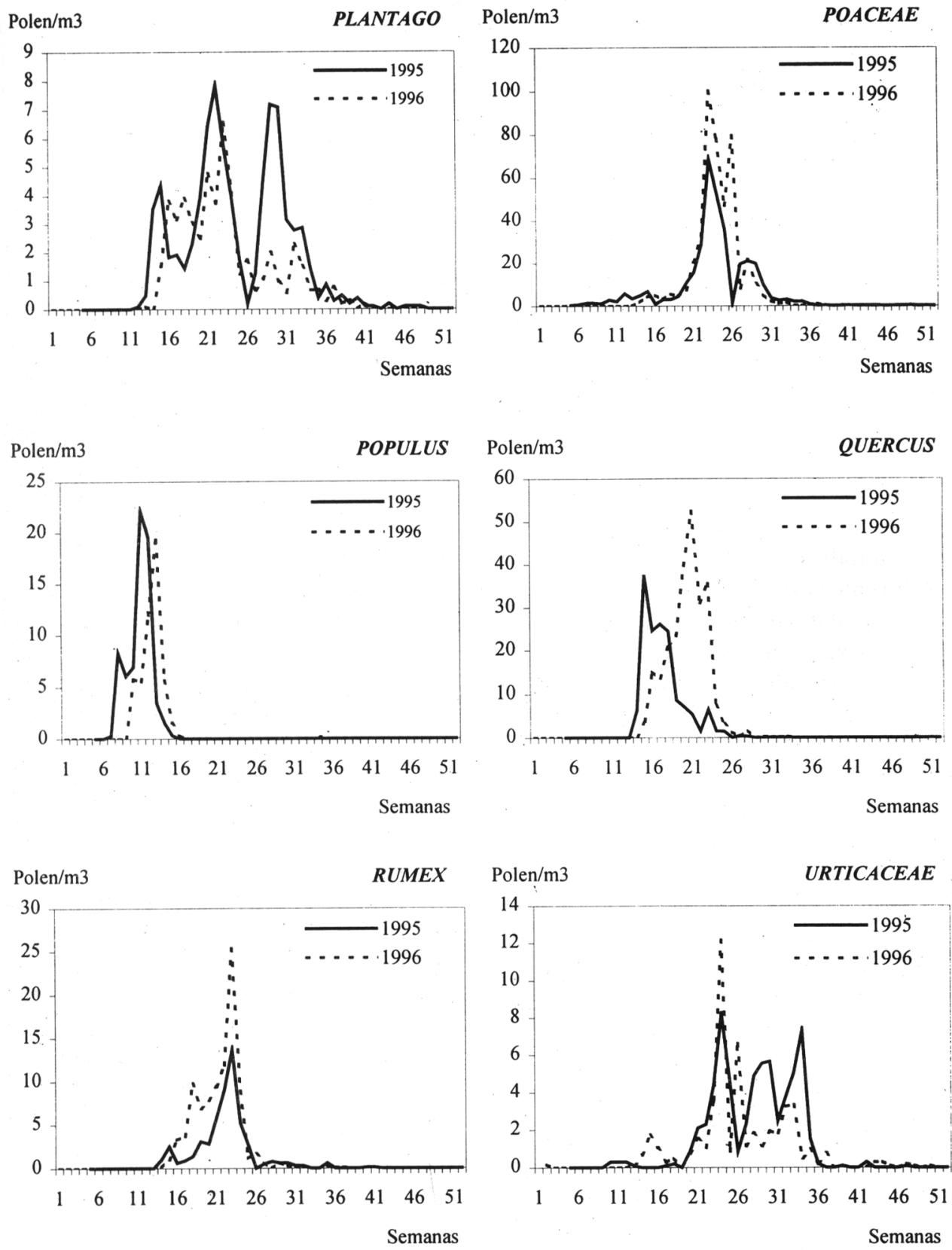

Figura 3. Evolución de las concentraciones medias semanales del polen de Plantago, Poaceae, Populus, Quercus, Rumex y Urticaceae, en la atmósfera de Ponferrada. Weekly average pollen concentration trend of the Plantago, Poaceae, Populus, Quercus, Rumex and Urticaceae in the atmosphere of Ponferrada. 
la ciudad. En la primavera, que es cuando se alcanzan niveles más altos, las concentraciones raramente superan los 30 granos $/ \mathrm{m}^{3}$.

La dinámica anual del polen de gramíneas puede observarse en la figura 3. Aunque es uno de los principales tipos polínicos, su porcentaje $(19,2)$ es inferior al de ciudades del interior de clima más continental como León (FernándezGonzález et al., 1998) o Palencia (Herrero \& Fraile, 1997). La gran cantidad de especies englobadas en esta familia hace que su polinización se prolongue durante mucho tiempo (Febrero-Octubre) con concentraciones máximas semanales de hasta 100 granos $/ \mathrm{m}^{3}$, alcanzada en la semana 23 de 1996.

El polen del castaño (Castanea sativa) es producido por los grandes ejemplares de este árbol, naturalizados en bosques próximos a la ciudad. Teniendo en cuenta que su polinización tiene lugar durante unas pocas semanas del periodo estival ( 7 semanas), este tipo polínico aparece en concentraciones elevadas en el mes de Junio (fig. 2), con valores máximos semanales de hasta $56 \mathrm{granos} / \mathrm{m}^{3}$. Las cantidades totales anuales son similares a los valores que Iglesias et al. (1998) y Rodríguez et al. (1998) encontraron en Orense y Vigo respectivamente.

Plantago (fig. 3), es un tipo polínico presente en la atmósfera durante un largo periodo de tiempo (de Abril a Septiembre). Sus concentraciones son bajas e inferiores a las recogidas en otras ciudades próximas como León (Fernández González et al., 1998), Palencia (Herrero \& Fraile, 1997) y Valladolid (Linares López et al., 1983).

Urticaceae (fig. 3) aparece en la atmósfera durante gran parte del año, pero en bajas concentraciones. Los valores máximos del mes de Junio, son debidos a la floración conjunta de Urtica y Parietaria.

Rumex (fig. 3) es otro de los tipos polínicos herbáceos descrito en los espectros polínicos de la atmósfera de la mayor parte de localidades de las regiones templadas, sus niveles más altos se alcanzan en los meses de Mayo y Junio, aunque su periodo polínico principal se prolonga desde mediados de Abril hasta finales de Julio.

\section{Características clínicas}

De los 139 casos estudiados, cuarenta y siete pacientes (34\%) tenían sensibilización a alergenos de interior, principalmente a ácaros del polvo; cuarenta y siete (34\%) mixta a alergenos de interior y pólenes y cuarenta y cinco $(32 \%)$ sólo a pólenes. De estos últimos, 18 individuos estaban monosensibilizados a gramíneas y 27 eran alérgicos a más de un grupo. Del total de pacientes observados, 84 $(60 \%)$ mostraron pruebas positivas a gramíneas, 38 pacientes $(27 \%)$ fueron positivos al polen de Plantago, aunque ninguno monosensibilizado. Y, en menor medida, se apreciaron reacciones positivas a Betula $(12 \%)$, Artemisia (8\%), Parietaria (7\%) y Chenopodium (7\%).

En el aspecto meramente clínico diremos que la mayoría de nuestros pacientes polínicos presentan síntomas en los meses de Mayo y Junio y, con menor intensidad, en Julio. A veces, refieren molestias a finales de Febrero y principios de Marzo como sucedió, por ejemplo, en 1994, fechas éstas en que hemos documentado la presencia de polen de gramíneas en cantidad significativa (Belmonte, J., comunicación personal). Por lo tanto pensamos que los pólenes de gramíneas son, con mucho, los que ocasionan más patología polínica en esta comarca.

\section{CONCLUSIONES}

Los dos primeros años de análisis polínico de la atmósfera de la ciudad de Ponferrada muestran que los pólenes de Cupressaceae, Poaceae, Pinaceae y Quercus son los más abundantes $(63,1 \%)$.

Se ha detectado un predominio anual de 
polen de especies leñosas frente a herbáceas. En general, los taxones arbóreos y arbustivos presentan periodos polínicos iguales o inferiores a 10 semanas, mientras que en los taxones herbáceos, el periodo polínico principal es superior a 20 semanas.

Entre los meses de Febrero y Julio se produce la máxima emisión de polen a la atmósfera de esta ciudad, mientras que en los meses de Octubre y Noviembre la cantidad polínica en el aire es prácticamente nula.

Desde el punto de vista clínico, los pólenes de Poaceae $(60 \%)$ son, con mucho, los que ocasionan mayor patología polínica, seguidos de Plantago $(27 \%)$ y Betula $(12 \%)$.

AGRADECIMIENTOS. Este trabajo ha sido financiado con cargo a un proyecto concedido por la Excma. Diputación Provincial de León. Queremos expresar nuestro reconocimiento al Excmo. Ayuntamiento de Ponferrada, que nos ha facilitado las dependencias y la instalación del captador polínico. Así como la colaboración desinteresada en la toma de muestras de los miembros del Laboratorio Municipal de Medio Ambiente, coordinados por A. Garnelo. Igualmente agradecemos a nuestros compañeros de Departamento: Emilio Puente y $\mathrm{M}^{\mathrm{a}}$ José López el habernos proporcionado la información sobre la vegetación autóctona de esta zona.

\section{BIBLIOGRAFÍA}

AIRA M. J., A. DOPAZO y F. J. RODRÍGUEZ -1998Aerobiología en Galicia: Estación de Santiago de Compostela (1995-1996). REA 3: 77-80.

BELMONTE, J. -1988- Concentración polínica en la atmósfera de Barcelona. Orsis 3: 67-75.

DAHL, A. \& S. STRANDHEDE - 1996- Predicting the intensity of the birch pollen season. Aerobiologia 12: $97-106$.

DOMÍNGUEZ VILCHES, E., C. GALÁN SOLDEVILLA, F. VILLAMANDOS DE LA TORRE \& F. INFANTE GARCÍA-PANTALEÓN -1991- Handling and evaluation of the data from the aerobiological sampling. Monografías REA/ EAN 1: 1-18.

EMBERLIN, J. C., J. NORRIS-HILL \& R. H. BRYANT -1990-A calendar for tree pollen in London. Grana 29: 301-309.
FERNÁNDEZ-GONZÁLEZ, D., R. M. VALENCIA BARRERA, A. M. VEGA MARAY y E. SAGÜÉS EMALDI -1998- Aerobiología en Castilla y León: Estación de León (1995-1996). REA 3: 49-52.

FRENGUELLI. G., F. Th.M. SPIEKSMA, E. BRICCHI, B. ROMANO, G. MINCIGRUCCI, S. A. H. NIKEL, W. DAN KAART \& F. FERRANTI -1991- The influence of air temperature of starting dates of the pollen season of Alnus and Populus. Grana 30: 196-200.

HERRERO, B. \& C. FRAILE - 1997- Annual variation of airborne pollen in the city of Palencia, Spain, 1990-92. Grana 36: 358-365.

IGLESIAS, I., J. MÉNDEZ y C. SEIJO -1998Aerobiología en Galicia: Estación de Ourense (1995-1996). REA 3: 73-76.

LINARES LÓPEZ, P. M., J. A. GÓMEZ CARRASCO y R. ANDIÓN DAPENA -1983- Estudio aerobiólogico mediante método volumétrico, de la atmósfera de Valladolid, durante los años 1981, 1982. En: N. Solé de Porta \& M. Suárez Cervera. Actas del IV Simposio de Palinología, Barcelona, 1982, 261-276. Ed. Univ. de Barcelona, Barcelona.

MORALES, J.; F. J. GONZÁLEZ MINERO, C. TOMÁS y P. CANDAU -1998- Análisis del contenido polínico de la atmósfera de Huelva. Lazaroa 19: 59-69.

NILSSON, S. \& S. PERSSON -1981- Three pollen espectra in the Stockholm region (Sweden) 1973-1980. Grana 20: 179-182.

RECIO, M., M. M. TRIGO, F. J. TORO y B. CABEZUDO -1996- Caracterización del contenido polínico invernal de la atmósfera de Málaga. Acta Bot. Malacitana 21: 65-70

RODRÍGUEZ, F. J., M. R. DÍAZ y V. JATO -1998Aerobiología en Galicia: Estación de Vigo (1995-1996). REA 3: 81-84.

TORO, F. J., M. M. TRIGO, M. RECIO y B. CABEZUDO -1997- Contenido polínico de la atmósfera de Estepona (Málaga): Año 1996. Acta Bot. Malacitana 22: 115-122.

TROISE, C., S. VOLTOLINI, G. DELBONO \& A.C. NEGRINI-1992-Allergy to pollen from Betulaceae and Corylaceae in a Mediterranean area (Genova, Italy). A ten-year retrospectyve study. J. Invest. Allergol. Clin. Immunol. 2(6): 313-317.

Aceptado para su publicación en Febrero de 1999

Dirección de los autores. A. VEGA MARAY, R. M. VALENCIA BARRERA y D. FERNÁNDEZGONZÁLEZ: Dpto. de Biología Vegetal. Universidad de León. Campus de Vegazana, s/n. 24071 León; J. M. ESTRADA: Unidad de Alergia. Complejo Hospitalario de León. 24071 León. 